\title{
The Influence of Cyclophosphamide on Immune Function of Murine Macrophages
}

\author{
Krzysztof Bryniarski \\ Jagiellonian University Medical College, \\ Department of Immunology, Krakow, \\ Poland
}

\section{Introduction}

\subsection{The structure of cyclophosphamide (CY) and its active metabolites acrolein (ACR) and phosphoramide mustard (PM)}

Cyclophosphamide (CY), an alkylating compound is commonly used as a cytoreductive agent in the treatment of cancer ( blood, breast, ovary) because of its ability to interfere with DNA synthesis and its pharmacological action on dividing cells (Ben Efraim 2001). Its action is however more complex since it exerts a strong influence on the immune system. Studies on cyclophosphamide are conducted for a long time, but its effect on macrophages (Mf) was not yet definite, therefore this study was attempted.

$\mathrm{CY}$ is in vitro inactive by itself, and is converted in vivo into two ultimately biologically active alkylating metabolites: phosphoramide mustard (PM) and acrolein (ACR).The first step of that complicated pathway of $\mathrm{CY}$ metabolism occurs in the liver and results in the formation of derivative hydroperoxycyclophosphamide and then it is followed by several enzymatic reactions that format carbonamide, aldehyde and carboxylacide inactive structures or lead to non-enzymatic formation of phosphoramide mustard and acrolein, active metabolites of cyclophosphamide which were tested in our immune research. Instead of highly unstable phosphoramide mustard (PM), we used nitrogen mustard (NM, mechlorethamine) (see Fig 1.) which is structurally and functionally related to PM, previously shown in vitro and in vivo to have the same activity as CY (Bryniarski et al. 1996).

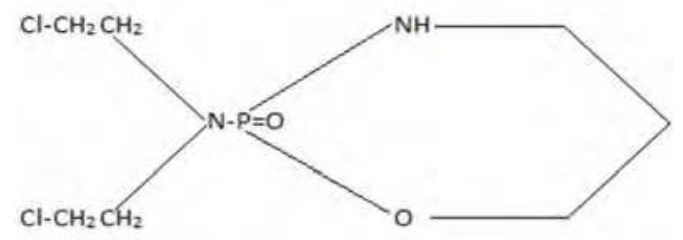

\section{CYCLOPHOSPHAMIDE}

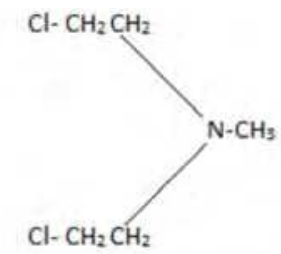

MECHLORETHAMINE

Fig. 1. The chemical structure of cyclophosphamide and nitrogen mustard 


\section{Differences of $\mathrm{CY}$ dose treatment used in the treatment of malignant diseases and autoimmune diseases in comparison to immune regulation}

Immunoregulatory function of CY is observed in doses $20-100 \mathrm{mg} / \mathrm{kg}$ in mice, which can be calculated in human treatment as below $2 \mathrm{mg} / \mathrm{kg}$ (less than $100 \mathrm{mg} / \mathrm{m}^{2}$ ). The higher doses of cyclophosphamide above $100 \mathrm{mg} / \mathrm{m}^{2}(2-3 \mathrm{mg} / \mathrm{kg})$ in humans mainly used in several pulses or as prolonged treatments are used in anticancer therapy of peripheral blood cancers and lymphoma (Audia et al. 2007; Vitolo et al. 2008) as well as in metastases of breast, ovarian and bronchial cancers (Snowden et al. 1997; Audia et al. 2007; Burger 2007) trophoblastic tumors (Cole et al. 2008), leiomyosarcoma (Durhan et al. 2009), pheochromocytoma (Adjalle et al. 2009), rhabdomyosarcoma (Breitfeld \& Mayer 2005).

Much higher doses of CY (50mg/ kg per 3 days) are proposed to be used in treatment of Graft Versus Host Disease (GVHD) after allogenic hematopoetic stem cells transplantation (alloHSCT) for treatment of hematologic malignancies (Luznik \& Fuchs 2010). Posttransplantation CY promotes tolerance in alloreactive host and donor T cells leading to suppression of both graft rejection and GVHD after alloHSCT.

In autoimmune diseases like sclerosis multiplex (MS), lupus nephritis or immune mediated neuropathies, CY is the medicament used for pulsed first treatment as well for the retreatment in several doses mainly higher than 100 up to $1600 \mathrm{mg} / \mathrm{m}^{2}$. It is proved that CY treatment seems to give better therapeutic effect in patients in earlier stages of MS where inflammation predominates over degenerative processes in the central nervous system (CNS). There is no evidence of efficacy in primary progressive MS or later stages of secondary progressive MS. In these high doses of CY therapy, patients show low proinflammatory cytokine secretion which activates anti-inflammatory cytokine secretion, what is suggested in an elegant review by Weiner and Cohen as one of curative effects in MS neurodegenerative disease (Weiner \& Cohen 2002). That high dose treatment can express quite a different suppressor effect on the immune system mediated by B and T lymphocytes that mediate two branches of humoral and cellular immune responses. High doses of $C Y$ used for the treatment of malignant or autoimmune diseases express more toxic effects on the immune system by elimination of different currently activated subpopulations of immune cells and lead to inhibition of inflammatory reactions which diminish the formation of degenerative lesions.

Cyclophosphamide is commonly used in multiagent chemotherapy rather than intravenous (i.v.) monotherapy for treatment of malignancies, therefore its dose can be relatively lesser than in case of single use (because of the effect of summarizing or multiplying of drug activity and toxicity). It also must be taken into consideration what kind of regulatory therapeutic effect is desired - the activating effect based on elimination of natural suppression in case of low doses treatment, or suppressor effect on the immune cells, maintained mainly by cytotoxic effect which appears in case of treatment with high doses of CY. It is estimated that the therapeutic dose of endoxan equals about $25-50 \%$ of toxic dose. The control of unwanted and unexpected toxic effects must be also taken into consideration in case of establishing any kind of CY therapy. Between the most frequent unwanted toxic effects observed in case of $\mathrm{CY}$ treatment is gonadal disfunction observed in man in a lower cumulative dose in man $\left(60 \mathrm{~g} / \mathrm{m}^{2}\right)$ less expressed in female (Vitolo et al. 2008). Moreover, apart from alopecia and nausea, the other toxic effects are infections and hemorrhagic cystitis. There is also an increased risk of cancer and bladder toxicity which appears in the 
cumulative life-time dose exceeding $80-100 \mathrm{~g}$ (which is about 50 doses of $1000 \mathrm{mg} / \mathrm{m}^{2}$ during life-time) (Weinar \& Cohen 2002) .

\section{Immunoregulatory activities of cyclophosphamide treatment - The evidence performed in mice by the low dose cyclophosphamide treated macrophages}

Macrophages (Mf) carry out the fundamental protective function of phagocyting and killing invading organisms and release a vast number of factors involved in host defense and inflammation. Moreover they play a critical role in the induction, regulation and expression of both cellular and humoral responses. These highly diversified functions are accompanied by heterogeneous morphology and biochemical and phenotypical characteristics.

\subsection{Methods for estimation of macrophage activity in innate and in adaptive humoral and cellular immune responses}

My experimental studies were aimed at the establishing the influence of low dose of $\mathrm{CY}$ treatment $(20-50 \mathrm{mg} / \mathrm{kg})$ on the macrophages (Mf) - the important cells involved in an innate and humoral or cellular adaptive immune responses in mice. The innate immune response was measured by both the capacity of reactive oxygen intermediates production and secretion by phagocytic activating macrophages and by the ability of production of proinflammatory cytokines (IL-1 $\beta$, IL-6 and TNF- $\alpha$ ) (Marcinkiewicz et al. 1994). The ability of cyclophosphamide treated $\mathrm{Mf}(\mathrm{MfCY})$ to secrete regulatory cytokine (proinflammatory IL-12 and inhibitory TGF- $\beta$ and IL-10) was also tested in their supernatant over the Mf culture and was estimated by ELISA (total concentration) (Bryniarski 2004 \& 2009), or in bioassays which utilize proliferation effect of particular cytokine by cytokine-dependent cell lines and measures exclusively bioactive form of particular cytokine (Marcinkiewicz et al. 1994 \& Bryniarski et al. 1996). The cell surface markers of tested Mf were estimated cytofluorimetrically in FACS (Szczepanik et al. 1993 \& Bryniarski et al. 2009), but the phenotypical differences between functionally differentiated macrophages were estimated by measuring the pattern of esterase activity (Czajkowska et al. 1995).

The induction of cellular response was tested in contact hypersensitivity against TNP/PCL hapten (Szczepanik et al. 1993, Bryniarski et al. 2004) which activates CD4 Th1 subpopulation of lymphocytes and macrophages and is classified by Pichler as a classical type IVa delayed type hypersensitivity (Lerch \& Pichler 2004; Posadas \& Pichler 2007). Humoral activity against corpuscular antigen was tested in plaque forming assay (PFA) from Mf pulsed with sheep red blood cells and cultured 4 days in the presence of naïve B cells. The antigen presenting activity was estimated as a number of plaque forming cells PFC/106 splenocytes (Bryniarski et al. 2004).

\subsection{Treatment of donors of macrophages with low dose of $\mathrm{CY}$ uncovers the subpopulation of peritoneal macrophages that induce CHS response in mice}

Application of hapten on the skin is a classical way to immunize for contact sensitivity reaction, but animals can also be sensitized by subcutaneous injection of hapten substituted peritoneal macrophages. However, if haptenated macrophages are injected i.v. a long-lasting unresponsiveness ensues, in which the activity of Th1 immune effector lymphocytes is 
obliterated by simultaneously recruited $\mathrm{CD}^{+}$suppressor cells. When subsequently skin sensitized, such animals have significantly diminished CHS reactions. Moreover this state of unresponsiveness can be adoptively transferred to naïve syngeneic animals by lymphoid cells.

The CHS reaction to trinitrophenyl (TNP/PCL) hapten is activated by CD4 Th1 lymphocytes which recognize TNP/PCL hapten on the surface of MHC class II expressed by hapten-labeled macrophages. After 5 days the sensitized recipients of TNP-Mf are able to reduce the CHS reaction when challenged by applying a very low dose of the same hapten (TNP/PCL) on the ear skin. The CHS reaction develops $24 \mathrm{~h}$ after challenge (with hapten) as ear swelling CHS response and is measured with the engineering micrometer and expressed in units of ear swelling (Szczepanik et al. 1993). When the donors of macrophages are injected intravenously (i.v.) with low dose of cyclophosphamide $(20-100 \mathrm{mg} / \mathrm{kg}$ ) a day before cell harvesting, then the cells are labeled with hapten and injected i.v. into naïve recipients instead of unresponsiveness strong contact hypersensitivity develops $24 \mathrm{~h}$ after the challenge with the same hapten. Moreover it was shown that in vivo CY treatment activates strong functional diversification of macrophage subpopulations in mice. Thus result of in vivo CY treatment, hapten-conjugated Mf (TNP-MfCY) when injected i.v. into naïve recipients TNP-MfCY activate strong contact hypersensitivity (CHS) reaction against hapten, instead of unresponsiveness induced by TNP-Mf mediated by CD8 ${ }^{+} \mathrm{T}$ suppressor lymphocytes, which inhibit hapten specific CHS response (Fig. 2).

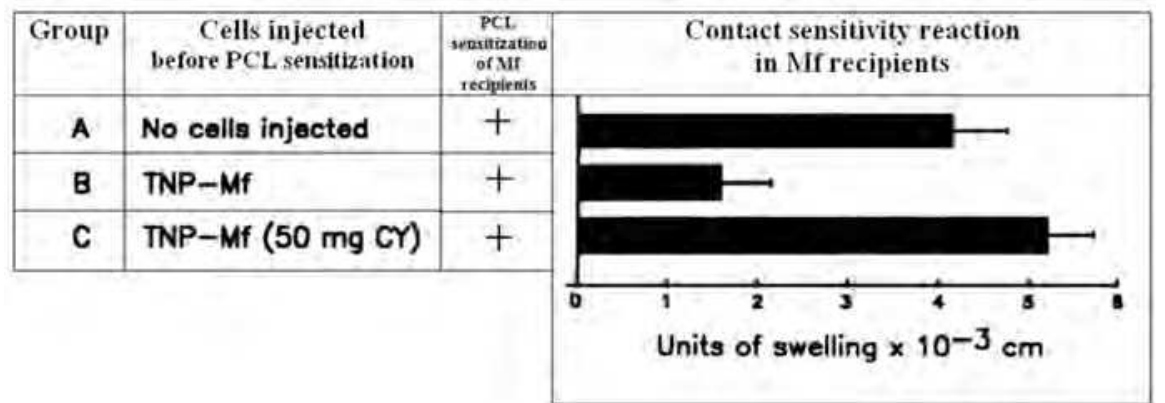

$\mathrm{CBA} / \mathrm{J}$ mice were injected i.v. with $1 \times 10^{6} \mathrm{TNP}-\mathrm{Mf}$ from donors treated (group C) or untreated (group B) with CY. Seven days later these animals and a group of naïve mice (group A) were skin sensitized with PCL and tested for contact hypersensitivity (CHS) after additional 4 days. Statistical significance group B ve groups A \& $\mathrm{C} p<0.002$

Fig. 2. Peritoneal macrophages from cyclophosphamide-treated mice do not induce suppressor cells.

It was shown by us that CY sensitive subpopulation of peritoneal macrophages responsible for induction of suppressor cells has higher density when separated in discontinuous gradient of Ficoll than population activating CHS response. Moreover it has also strong adherent and higher phagocyting properties and has high expression of Fc $\gamma$ RI and Fc $\gamma$ RII $(33-44 \%)$ in comparison to the low density cell fraction, which is $\mathrm{CY}$ resistant. The latest fraction of cells is weakly phagocytic and adherent, has less Fc $\gamma R$ (22-26\%). It is widely known that antigen presenting cells (APC) are macrophages, dendritic cells and B lymphocytes. First two populations can phagocyte, but last is inactive in this activity. For two reasons it seems unlikely that previously described light fraction of cells are contaminated with dendritic cells. 
First almost all of them labeled with macrophage specific F4/80 antibody, second, as shown previously by Steinman and Cohn peritoneal exudate cells induced by thioglycolate, although contaminated by several cell types do not contain dendritic cells (Szczepanik et al. 1993). Both subpopulations of tested macrophages express no significant differences in Mac-1 (30-50\%) and Mac-3 (33-46\%) markers and no differences were found between CY-treated and untreated mice in a surface expression of I-A (7-14\%) and I-E (3-6\%) antigens. We were not able to associate the differences in surface markers expression in both populations of macrophages with variety of their bioactivity and immune function (Szczepanik et al. 1993). We also found that Mf CY express slightly higher percentage of molecules important in the stimulation of phagocytosis (CD14 and CD23) and molecules basic for activation of antigenpresenting immune response (CD80/CD86 and MHC class II) in comparison to control oilinduced peritoneal macrophages. Results are shown in Table 1.

\begin{tabular}{|c|c|c|c|c|c|}
\hline Macrophages & CD23/Mac3 & CD80/Mac3 & CD86/Mac3 & CD14/Mac3 & DR/Mac3 \\
\hline Mf & 62.0 & 50.0 & 51.6 & 59.3 & 45.4 \\
\hline $\begin{array}{c}\text { Mf CY } \\
{[50 \mathrm{mg} / \mathrm{kg}]}\end{array}$ & 73.7 & 68.4 & 71.6 & 71.7 & 59.0 \\
\hline
\end{tabular}

Oil-induced macrophages were labeled for direct immunofluorescence with monoclonal antibodies specific against specific macrophage antigen (Mac3-FITC) and cell surface markers (CD23-PE, CD80PE, CD86-PE, CD14-PE, MHCII DR-PE). Results are expressed as a percent of double positive cells. The measurement was performed on the FASC Cytoron-Absolute.

Table 1. Expression of selected surface markers on macrophages isolated from oil-induced peritoneal cavity from donors treated or not treated with cyclophosphamide in dose 50 $\mathrm{mg} / \mathrm{kg}$.

In following experiment we have shown (Bryniarski et al. 2009) that cyclophosphamide in vivo as well as its both tested metabolic products in vitro - acrolein (ACR) and mechloretamine (NM) (nitrogen mustard - an analogue of phosphoramide mustard) make TNP-substituted Mf (TNP-Mf) immunogenic for the induction of CHS response. The results are shown in Table 2.

\begin{tabular}{|c|c|c|}
\hline Group & Mice injected with & CHS response [U x 10-2 mm] \pm SD \\
\hline A & TNP-Mf & $0.2 \pm 0.84$ \\
\hline B & TNP-Mf CY & $4.8 \pm 0.68$ \\
\hline C & TNP-Mf ACR & $5.3 \pm 1.12$ \\
\hline D & TNP-Mf NM & $5.2 \pm 0.66$ \\
\hline E & TNP-Mf ACR \& NM & $5.5 \pm 0.90$ \\
\hline
\end{tabular}

The following abbreviations are used: TNP-Mf - TNP substituted Mf from control mice; TNP-Mf CY Mf from mice treated with CY (50 mg/ kg); TNP-Mf ACR - normal Mf treated in vitro with $10^{-7} \mathrm{M} \mathrm{ACR;}$ TNP-Mf NM - normal Mf treated in vitro with 10-7 M ACR; TNP-Mf ACR \& NM - Mf treated with both metabolites.

CBA/J mice were injected i.v. with $1 \times 10^{6} \mathrm{TNP}$-substituted Mf . Seven days after TNP-Mf injection the mice were tested for CHS reaction which is expressed in units $\times 10^{-2} \mathrm{~mm} \pm \mathrm{SD}$. The negative values (ear swelling in control, unimmunized mice) were substracted from experimental values. The statistical significance (a posteriori Bonferroni test): Group A vs. Groups B, C, D, E p<0.001. Each group consisted of five mice.

Table 2. Immunognecity of oil-induced peritoneal macrophages untreated or treated in vivo with $50 \mathrm{mg} / \mathrm{kg} \mathrm{CY}$ or in vitro with acrolein (ACR) or mechloretamine (NM). 


\subsection{The phenotypical differences between functionally differentiated macrophages estimation of activity IL- 6 and a heterogeneity of $\alpha$ - and $\beta$ - naphtyl acetate esterase isoenzymes}

Some differences were shown when a population of different immune functions were tested by unspecific esterase activity, which is regarded to play a role in intracellular processing and trafficking of antigen (Czajkowska et al. 1995). In these experimental works we tested two different, but phenotypically indistinguishable macrophage clones 59 and 63 obtained from Martin Dorf and coworkers from Harvard University, Boston, MA. Both are adherent and phagocytic, produce IL-1 and IL-6 and constitutively express a number of identical cell surface markers, including Ia. However they differ functionally and while clone 59 presents antigen to Th1 lymphocytes, clone 63 induces suppressor T lymphocytes. These obvious functional differences in antigen presenting capacities between the seemingly phenotypically identical cell lines were identified by us in testing them for activity of nonspecific $\alpha$ - and $\beta$ - naphtyl acetate esterase isoenzymes and secretion of IL-6 in case of cell lines stimulated with mechloretamine (NM)- the alkylating agent relating to cyclophosphamide. The isoenzymatic patterns of $\alpha$-esterase express strong differences in line 59 treated with $\mathrm{MN}$ in comparison to background non-activated cells. In line 59, NM treatment increases the heterogeneity and activity of esterase in both $\mathrm{pH} 7.5$ and $\mathrm{pH}$ 5.8. In contrast to line 59 no differences in the isoenzymatic pattern of $\alpha$-esterase in NM-treated and untreated 63 line cells were found. The $\beta$-esterase activity was also tested but no significant differences were found between untreated and MNtreated cell lines.

The results in Table 3 show that CHS inducing clone 59 produces little IL-6 and NM does not activate its production in comparison to basal activity. In contrary clone 63 is a high producer of IL-6 and stimulated by NM.

\begin{tabular}{|c|c|c|}
\hline \multirow{2}{*}{ Groups } & $\begin{array}{c}\text { Clone 59 } \\
\text { IL-6 }[\mathrm{ng} / \mathrm{mL}] \mathrm{X} \pm \mathrm{SD}\end{array}$ & $\begin{array}{c}\text { Clone 63 } \\
\text { IL-6 }[\mathrm{ng} / \mathrm{mL}] \mathrm{X} \pm \mathrm{SD}\end{array}$ \\
\hline Mf & $62 \pm 9.6$ & $111 \pm 9.3$ \\
\hline Mf \& NM 10-6 M & $27 \pm 4.3$ & $358 \pm 3.1$ \\
\hline Mf \& NM 10-8 M & $35 \pm 4.7$ & $226 \pm 1.5$ \\
\hline Mf \& NM 10-10 M & $21 \pm 5.5$ & $209 \pm 2.1$ \\
\hline
\end{tabular}

$2 \times 10^{7} \mathrm{Mf}$ were treated with $\mathrm{NM}$ at concentrations from $10^{-10}$ to $10^{-6} \mathrm{M}$ for 40 min at $0^{\circ} \mathrm{C}$ then thoroughly washed out from NM with phosphate buffered saline (PBS). Cells cultured in RPMI 1640 medium supplemented with 5\% fetal calf serum (FCS) at concentration $10^{6} \mathrm{Mf}$ per $\mathrm{mL}$ in 24 wells flat bottom plates at $37{ }^{\circ} \mathrm{C}$ at $5 \% \mathrm{CO}_{2}$. The concentration of IL-6 was estimated in bioassay with B9 cell line with (data not shown, but all results were estimated less than $2 \mathrm{ng} / \mathrm{mL}$ ) or without $\mathrm{mAb}$ anti-IL-6 and expressed in $\mathrm{ng} / \mathrm{mL}$.

Table 3. The production of IL-6 by Mf cell line 59 and 63 treated with different concentration of nitrogen mustard

Our experiments show that two macrophage lines differ in isoenzyme patterns such that $\alpha$ and $\beta$ esterases of line 59 which induces immunity, are more heterogeneous than esterase of line 63, which induces suppression. Both cell lines, when non activated with NM however 
produce comparable amounts of IL-6 (Czajkowska et al. 1995). We have shown previously the peritoneal macrophages (Bryniarski et al. 1996), like line 63 cells, when tagged with the antigen, induce $\mathrm{T}$ suppressor cells, however, when treated with $\mathrm{NM}$, a pharmacological derivative of cyclophosphamide, produce high amounts of IL-6, also change their functional (suppressive) properties to activation the hapten specific CHS immune response (Bryniarski et al. 2009).

It was also shown that NM, changed the isoenzyme pattern in line 59, which was accompanied by somewhat decreased production of IL-6, while esterases of line 63 were not affected by NM, which however activated the production of IL-6. NM, like other alkylating factors, binds covalently and non-selectively to variety of molecules including amino acids, proteins and DNA. Since the Mf were incubated with $\mathrm{NM}$ at $0^{\circ} \mathrm{C}$ the possibility of its intracellular penetration was negligible and we presume that under such condition NM binds mainly to cell surface proteins. We suggested that the translation of intracellular membrane signal acts in a different way, which can result in a different production of IL-6 by line 59 and 63 cells and their isoenzyme patterns after NM stimulation (Czajkowska et al. 1995).

\subsection{Proinflammatory cytokine secretion by functionally different subpopulation of Mf treated with CY or its derivatives ACR or NM}

CY influence on the cytokine releasing activity by the functionally different subpopulation of macrophages was examined by Marcinkiewicz and Bryniarski (Marcinkiewicz et al. 1994 and Bryniarski et al. 1996 \& 2009). They have shown that low dose of $\mathrm{CY}$ treatment activates Mf to production and secretion of mainly IL-6 while simultaneously diminish TNF- $\alpha$ and IL-1 $\beta$ production (Marcinkiewicz et al. 1994). The strong activation of IL-6 production was observed in case of peritoneal macrophage stimulation with mechloretamine (NM) the analogue of nitrogen mustard (Bryniarski et al. 1996). This is a common phenomenon which is observed in NM-treated oil-induced and thioglycolate peritoneal macrophages obtained from different mouse strains (CBA/J, Balb c, C57/BL6 and SWISS) and tested in bioassay with IL-6 dependent B9 cells as well as in IL-6 ELISA assay (Bryniarski et al. 1996 \& 2009).

\subsubsection{Production of cytokines by control Mf or Mf from animals treated in vivo with CY or Mf treated in vitro by its metabolites}

Control Mf or Mf from CY-treated animals $(50 \mathrm{mg} / \mathrm{kg}$ ) or Mf incubated in vitro with ACR $\left(10^{-7} \mathrm{M}\right)$ or NM $\left(10^{-6} \mathrm{M}\right)$ were cultured for 24 or $48 \mathrm{~h}$ in RPMI 1640 medium and the production of five different cytokines was measured in the resultant supernatants by ELISA. These results are shown in Table 4. Mf from CY treated animals (Group B) and Mf treated in vitro with NM (Group D) showed an increased production of pro-inflammatory IL-6 and IL12 , and a decreased production of anti-inflammatory IL-10 and TGF- $\beta$ cytokines compared to the control group (Group A). Mf treated with ACR (Group C) manufactured more IL-6 and less TGF- $\beta$ than control cells (Group A), but the production of IL-12 remained unchanged. Treatment with $\mathrm{CY}$ or its derivatives did not influence TNF- $\alpha$ production although in Group B, there it was somewhat lower than in the control group. 


\begin{tabular}{|c|c||c|c|c|c|c|}
\hline \multicolumn{2}{|c||}{ Cell cultured } & \multicolumn{5}{c|}{ Cytokine production [pg/ml] } \\
\cline { 3 - 7 } & & TNF-a & IL-6 & IL-10 & IL-12 & TGF- $\beta$ \\
\hline \hline A & Mf & $250 \pm 5$ & $283 \pm 7$ & $61 \pm 3$ & $213 \pm 3$ & $109 \pm 2$ \\
\hline B & Mf CY [50 mg/kg] & $182 \pm 4$ & $467 \pm 4$ & $22 \pm 4$ & $455 \pm 14$ & $50 \pm 4$ \\
\hline C & Mf ACR [10-6 M] & $287 \pm 21$ & $982 \pm 25$ & $33 \pm 2$ & $167 \pm 10$ & $33 \pm 3$ \\
\hline D & Mf NM $\left[10^{-7} \mathrm{M}\right]$ & $245 \pm 30$ & $1500 \pm 100$ & $47 \pm 4$ & $460 \pm 28$ & $59 \pm 17$ \\
\hline
\end{tabular}

Five $\times 10^{5}$ control Mf or cells from cyclophosphamide-treated animals (Mf CY) or Mf treated with acrolein (Mf ACR) or nitrogen mustard (Mf NM) in vitro (for details see legend to Table 3 ) were cultured in $1 \mathrm{ml}$ of RPMI 1640 medium supplemented with 5\% FCS for 24h (TNF-a and IL-6) or 48h (other cytokines) and concentrations of cytokines were measured by ELISA assays. Table 2 shows the results of one representative experiment out of three as the mean of three estimations \pm SD.

Table 4. Cytokine production by macrophages (Mf) from naïve mice, or animals treated with cyclophosphamide (CY) in vivo or acrolein (Mf ACR) or nitrogen mustard (Mf NM) in vitro.

\subsubsection{High tolerogenicity of TNP-Mf in vivo can be reversed by administration into recipients of anti-IL-10 and/or anti-TGF- $\beta$ mAbs}

Since inefficient immunogenicity of administered intravenously control TNP-Mf as compared with CY-treated cells may be due to the different cytokine sets that they produce, we injected these cells into recipients that simultaneously received an i.v. injection of $500 \mu \mathrm{g}$ of anti-IL-10, anti-TGF- $\beta$ or a mixture of both antibodies. The CHS reaction was measured 7 days later. Figure 3 shows that TNP-Mf

\begin{tabular}{|c|c|c|c|c|}
\hline \multirow[t]{2}{*}{ Group } & \multicolumn{2}{|c|}{$\begin{array}{l}\text { Treatment of recipients } \\
\text { with monoclonal antibody }\end{array}$} & \multirow{2}{*}{$\begin{array}{c}\text { Cyclophosphamide } \\
\text { treatment of } \\
\text { donors of MIf }\end{array}$} & \multirow[t]{2}{*}{$\begin{array}{l}\text { Contact sensitivity response } \\
\qquad( \pm \text { SD })\end{array}$} \\
\hline & $\mathrm{mAb} a \mathrm{Ll}-10$ & mAb $\alpha$ TGF- $\beta$ & & \\
\hline A & - & - & - & 一 \\
\hline B & - & - & + & \\
\hline C & + & - & - & \\
\hline D & - & + & - & \\
\hline E & + & - & + & \\
\hline F & + & + & - & \\
\hline G & + & + & + & \\
\hline & & & & Units of ear swelling $\left(1 \theta^{-2} \mathrm{~mm}\right)$ \\
\hline
\end{tabular}

CBA/J mice were injected i.v. with $1 \times 10^{6} \mathrm{TNP}$ substituted Mf (groups A, C, D and F) or TNP-Mf CY (groups B, E, G). In the groups $\mathrm{C}$ and $\mathrm{E}$ the mice simultaneously received i.v. $500 \mu \mathrm{g}$ of anti-IL-10 mAb and in group $\mathrm{D}, 500 \mu \mathrm{g}$ anti-TGF $\beta \mathrm{mAb}$, and in groups $\mathrm{F} \& \mathrm{G}$ both $\mathrm{mAbs}$ were given. Seven days later the $\mathrm{CHS}$ response was measured (see legend to Table 2). Statistiacally significant (a posteriori Bonferroni test) Group A ve groups B, C and F p<0.002; group A ve groups E and G p<0.001; Group B ve groups $\mathrm{C}$, F and $\mathrm{G} \rightarrow \mathrm{NS}$; group $\mathrm{B}$ ve $\mathrm{D} \mathrm{p}<0.02$

Fig. 3. Tolerance induced by intravenous injection of TNP-Mf can be reversed by simultaneous administration of anti-IL-10 and/or anti-TGF- $\beta$ mAbs. Comparison with in vivo $\mathrm{CY}$ treatment $(50 \mathrm{mg} / \mathrm{kg}$ ) of TNP-Mf (TNP-Mf CY) combined with administration with mAbs anti-IL10 or/and anti-TGF- $\beta$. 
obtained from naïve donors were non-immunogenic (Group A) but from CY-treated donors (Group B) induced CHS response, while animals treated with anti-IL-10 alone (Group C \& E) or together with anti-TGF- $\beta$ (Group F \& G) from both donors produced a significant CHS reaction. Anti-TGF- $\beta$ alone (Group D) had no influence on the level of the CS reaction.

\subsection{Production of the reactive oxygen intermediates by Mf CY or macrophages treated with low doses of ACR or NM}

The testing of innate immune response mediated by macrophages can be estimated by reactive oxygen intermediates (ROI's) production. We use the luminol-dependent chemiluminescence as a measure of activity in Mf stimulated by opsonized zymosan particles. Figure 4 shows that Mf from animals treated with 20 or $50 \mathrm{mg} / \mathrm{kg}$ of CY produce a significantly increased level of ROI's while the dose of $100 \mathrm{mg} / \mathrm{kg}$ remains without effect and $200 \mathrm{mg} / \mathrm{kg}$ diminished the production of ROI's below the control level. A marked increase of ROI's level was induced by in vitro incubation Mf in a low concentration of NM $\left(10^{-7}\right.$ or $10^{-6} \mathrm{M}$ ) while $10^{-5} \mathrm{M}$ had a slightly inhibitory effect. ACR at concentration $10^{-7} \mathrm{M}$ and $10^{-6} \mathrm{M}$ had no effect when compared with control $\mathrm{Mf}$, whereas $10^{-5} \mathrm{M}$ was inhibitory.
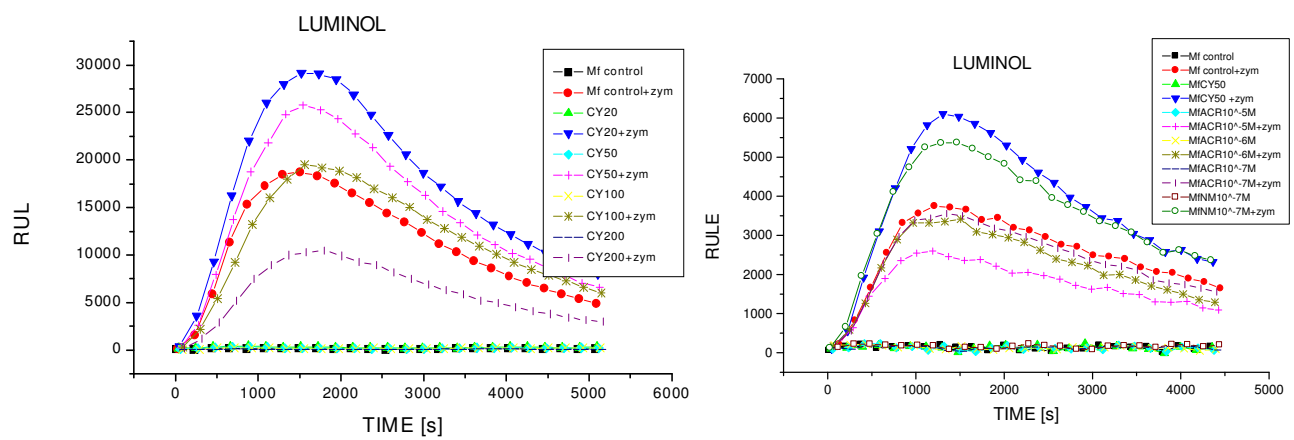

Production of reactive oxygen intermediates (ROI's) by macrophages is dependent upon the dose of cyclophosphamide (CY) used to treat donors. The highest activity is observed with doses $20-50 \mathrm{mg} / \mathrm{kg}$. The higher dose of CY (200 mg/kg) decreases ROI's production by Mf in comparison with control cells (Mf). The low concentration of nitrogen mustard (NM) used to treat Mf increases the production of ROI's to a similar level as in vivo treatment of Mf-donors with CY $(50 \mathrm{mg} / \mathrm{kg})$. Treatment by $10^{-5} \mathrm{M}$ NM was slightly inhibitory (results not shown). Treatment of Mf with acrolein (ACR) $\left(10^{-5}-10^{-7} \mathrm{M}\right)$ reduces the secretion of ROI's in a dose dependent manner in comparison to the control group (Mf). Zym - zymosan

Fig. 4. Influence of in vivo treatment with cyclophosphamide $(\mathrm{CY})$ and in vitro treatment with nitrogen mustard or acrolein on the production of reactive oxygen intermediates (ROI's)by murine peritoneal Mf.

\subsection{Testicular macrophages (TMf) and their immune response in the reaction with CY}

Residual testicular macrophages (TMf) that are present in interstitial tissue of male gonads are regarded as essential cells for male reproductive function involved in the regulation of hormonal balance in the testis. TMf by released products and also directly by cell-to-cell contacts participate in the process of steroidogenesis by Leydig cells. They also influence the behavior of Sertoli cells by releasing mediators and regulate spermatogenesis in testis. 
Apart from that function TMf play an important role in their functional contribution of anatomical blood-testis barrier formed by Sertoli cells (Bryniarski et al 2004).

\subsubsection{Induction of contact hypersensitivity by TMf and effect of CY-treatment}

Similarly to peritoneal macrophages testicular macrophages can be purified by glass adherence or fractionation on discontinuous gradient (Bryniarski 2004). Results in Table 5 show that TNP substituted TMf (TNP-TMf) when injected i.v. into naïve recipient induce unresponsiveness since the following PCL skin sensitization fail to induce CHS reaction (group B), but TNP-TMf obtained from donors treated with low dose of CY under the seen conditions express strong CHS reaction comparable with control mice actively immunized with PCL (group C ve group A).

\begin{tabular}{|c|c|c|c|}
\hline Groups & $\begin{array}{c}\text { Cells injected i.v. before } \\
\text { PCL sensitization }\end{array}$ & $\begin{array}{c}\text { PCL } \\
\text { sensitization }\end{array}$ & $\begin{array}{c}\text { CHS reaction in TMf } \\
\text { recipients in units of swelling } \\
\times 10^{-2} \mathrm{~mm}\end{array}$ \\
\hline A & No cells injected & + & $8.2 \pm 1.25$ \\
\hline B & TNP-TMf & + & $2.5 \pm 0.80$ \\
\hline C & TNP-TMf $(50 \mathrm{mg} \mathrm{CY})$ & + & $7.5 \pm 1.54$ \\
\hline
\end{tabular}

Purified TMf obtained from CY-untreated (group B) or CY-treated donors (group B) when substituted with TNP hapten (TNP-TMf) were injected i.v. into mice. Seven days later all TMf recipients and naïve mice (group A) were skin sensitized with 5\% PCL and 4 days later challenged on ear skin with $0.4 \%$ PCL. $24 \mathrm{~h}$ later CHS ear swelling response was measured with engineers micrometer and results expressed in units of swelling $\times 10^{-2} \mathrm{~mm}$. Statistics: group A ve group $B \mathrm{p}<0.001$, group $B$ ve group $C \mathrm{p}<0.01$.

Table 5. Testicular macrophages (TMf) from cyclophosphamide-treated mice do not induce suppressor cells in contrast to non-CY treated donors.

Our further experiments show that the tolerogenic activity of TNP-TMf is mediated by the high cytokine secretory activity mainly TGF- $\beta$. This cytokine is a very basic tool for functional strategy of TMf within testis and generates the state of organ as immune privileged site.

The methods used by us for obtaining enriched populations of TMf form mixture of testicular interstitial cells lead to separation of TMf into two functionally different cellular fractions - low density (fractions between interfaces of Percoll gradient 21/27 - 33/39) and high density (over 39/45). Low density fraction produces significantly more TGF- $\beta$ than heavier cells and are CY-sensitive, while high density cells are not (Bryniarski et al. 2004). Our later experiments show that elimination of TGF- $\beta$ activity by injection of anti-TGF- $\beta$ $\mathrm{mAb}$, but not anti-IL-10 mAb completely removed unresponsiveness obtained in TNP-TMf recipients after i.v. injection. The other adequate results implementing experiments with anti-TGF- $\beta$ mAbs are shown in our paper (Bryniarski 2004).

Our experiment with TMf shows that injected intravenously induced CHS response in recipients pre-treated with $\mathrm{CY}$. We found them also actively presenting corpuscular antigen (SRBC) in humoral response (Bryniarski et al. 2004). Again the low density subpopulation of TMf failed to induce CHS when injected i.v. into recipients, but in fact induced the state of 
tolerance in which subsequent skin application of hapten did not lead to development of contact sensitization. More interestingly, pretreatment of TMf donors with CY made the whole TMf population immunogenic. The mechanisms of action of $\mathrm{CY}$ are not cleared, but it has been argued that $\mathrm{CY}$ metabolites bind to sulfhydryl groups on antigen presenting cells (APC) changing their function (Bryniarski et al. 2004).

\section{Discussion}

Our results show that cyclophosphamide in vivo and both its metabolic highly reactive alkylating products - $\alpha \beta$-unsaturated aldehyde acrolein (ACR) and nitrogen mustard a derivative of phosphoramide mustard second metabolic agent formed in CY metabolism, activate TNP substituted Mf that leads to activation of CHS reaction mediated by Mf and hapten specific Th1 lymphocytes. We are tempted to suggest that this activity is mediated by the net of different proinflammatory (TNF- $\alpha$, IL-1 $\beta$, IL-6) and suppressory (IL-10 and TGF- $\beta$ ) cytokines secreted by Mf which can uncovering TNP-specific immunization activated by TNP substituted Mf and change their potential from inhibition of unresponsiveness (untreated Mf) into Mf immunogenicity (treated with CY or with CY metabolites).

\subsection{CY and its action on CHS}

Low dose of CY activates mainly of subpopulation of oil- or thioglycolate- induced peritoneal macrophages which are able to present hapten and subsequently activate trigger of Th1 mediated immune CHS response and diminishes the bioactivity of high density subpopulation of macrophages. That subpopulation induces specific immunologic unresponsiveness as a result of activating a network excess of efferent suppressor cells and mainly cooperates with $\mathrm{T}$ suppressor $\mathrm{CD}^{+}$hapten specific cells (Treg) and mediates the hapten specific tolerance. (Szczepanik et al. 1993; Marcinkiewicz et al. 1994, Bryniarski et al. $2004 \& 2009$ ). The question arises as to how treatment with ACR or NM converts tolerogenic Mf into immunogenic Mf. One possibility is that these CY metabolites disrupt the function of the Mf subpopulation that induces Treg cells. Alternatively they can enhance the activity of Mf subpopulation responsible for the induction of Th1 cells that mediates CHS reaction. Finally these two possibilities are not mutually exclusive and the increased production of IL12 and IL-6, and the simultaneously decreased production of anti-inflammatory IL-10 and TGF- $\beta$ cytokines, make these later assumptions most likely.

\subsection{CY regulates cytokine network released by $\mathrm{Mf}$}

One of the most strongly expressed of $\mathrm{CY}$ and their derivatives treatment of macrophages is the activation of IL-6 production (Bryniarski 1996, 2009) . Our results do not address directly the questions by which mechanisms NM modifies the macrophages to produce more IL-6. IL-6 gene expression can be induced by a variety of physiological (cytokines, growth factor, bacterial products) and non-physiological stimuli (certain toxins, medicaments, prostaglandin E1), by at least three different signals pathways (diacyloglycerol, cAMP- and $\mathrm{Ca}^{2+}$ activated pathways). At the DNA level three functional promoter domains were described in conserved region of IL-6 promoter (MRE, NF-IL6 and NFkB). Exactly how transduction pathways are assigned seems unknown with the possible exception of protein kinase C signal which seems to focus on MRE region (Bryniarski et al. 1996). Since NM 
binds to both DNA and proteins, it could stimulate IL-6 production directly by alkylating any domain of promoter region, or indirectly, by the alkylation of cell surfaces or by both mechanisms simultaneously.

IL-6 is one of the major mediators of the immune response, with pleotropic and sometimes opposed effects on many different targets. It has been shown for instance that IL-6 enhances the cytotoxic activity of NK cells, thus may potentially augment the host defenses and contribute to anti-tumor effects of alkylating agents. Nonetheless, in case of the IL-6 dependent tumours like myelomas or plasma cell leukemias increased IL- 6 level could be deleterious. The increased production of IL-6 may be responsible for observed paradoxical effects of $\mathrm{CY}$ which under certain conditions enhances, rather than suppresses both the humoral and cell mediated immune responses (Bryniarski et al.1996).

Apart from the influence of $\mathrm{CY}$ and its metabolites on the IL-6 production the inhibition of IL-10 and TGF- $\beta$ production by macrophages was also observed. The results presented above in Table 4 and Fig. 3 clearly suggest that the state of tolerance or unresponsiveness observed after TNP-Mf i.v. injection seems to be mediated by the network of pro- and antiinflammatory cytokines secreted from macrophages and also tentatively delivered by natural regulatory cells. It is highly unlikely that low concentration of cytokine metabolites $\left(10^{-7}\right.$ and $\left.10^{-6} \mathrm{M}\right)$ have a direct cytotoxic effect on the Treg-inducing Mf since the cell viability remains unchanged during $24 \mathrm{~h}$ culture. Our interpretation is also supported by finding that shifting the balance between pro- and anti-inflammatory cytokines allows for deliberate manipulation of the outgoing response. IL-10 and TGF- $\beta$, which are anti-inflammatory cytokines, inhibit the activity of Th1 cells and Mf and down-regulate their function. As shown in Figure 3, administration of anti-IL-10 and/or anti-TGF- $\beta$ mAbs into animals which received non-immunogenic TNP-Mf restores their immune potential although to different degrees. It indicates that the key suppressive cytokine is IL-10, a finding that is supported by other groups (Bryniarski et al. 2009). In a symmetrical situation, as we have shown previously, administration of anti-IL-12 antibodies inhibits the function of immunogenic TNP-Mf in vivo (Bryniarski et al. 2009). The increased production of IL-12 and IL-6 by macrophages indicates that the cell surface signal delivered by ACR or NM activates the transcription factor NF- $\mathrm{kB}$ required for the release of inflammatory cytokines (Bryniarski et al. 2009). As reported by other groups, ACR, when allowed free access to the interior of the cell, can either block or enhance the activity of NF- $\kappa B$ in alveolar macrophages depending on the design of cell treatment (Bryniarski et al. 2009).

\subsection{CY modulates oxygen radicals formation by macrophages}

Our results indicate that $C Y$ upregulates not only the specific immune response, by converting non-immunogenic (tolerogenic) Mf into antigen-presenting cells but also positively influences a typical parameter of innate immunity - production of oxygen radicals. In up-regulating the immune function of Mf, ACR and NM had much the same effect. This was however not the case with regard to the production of ROI's by these cells. Using the low concentrations of metabolites, NM was highly stimulatory while ACR did not influence the formation of oxygen radicals above the level observed in control Mf (high concentration of both metabolites were inhibitory). One possibility is that the ACR and NM bind to different targets on the cell surface. NADPH oxidase catalyzing the generation of ROI is composed of several cytosolic and membrane-bound proteins which, after the cell 
receives a proper signal (e.g. phagocytosis), translocate to form an active enzyme. We propose a possible explanation that under our experimental conditions, $A C R$, in contrast to $\mathrm{NM}$, does not bind efficiently to important docking proteins to trigger the increased production of ROI. Conflicting results regarding ROI production were also published by other groups. Some reports describe the inhibitory activity of ACR, and others indicate an increased production of radicals. In effect, one can conclude that experimental conditions were the key (Bryniarski at al. 2009).

Our experimental data showed that untreated and in vivo $C Y$ treated populations of peritoneal macrophages produce the similar level of nitrogen oxide (Marcinkiewicz et al. 1994) which does not allow to speculate on its function in the immune regulatory system mediated by macrophage stimulated with $\mathrm{CY}$.

\subsection{Immunomodulation in chemotherapy with low doses of CY}

Our data show that chemotherapy by $\mathrm{CY}$ or its products may activate the immune system by modulating cytokine networks and activation of Mf. This may lead to an enhancement of antigen-specific cell mediated immunity but also to activation of mechanisms of innate immunity mediated by Mf, like the production of ROI. Additionally, in animal models derivatives of different mustards led to decreased secretion of IL-10 and TGF- $\beta$ by tumor cells and to their elimination. These and other similar experiments in humans support the notion that, at a correct dosage, $\mathrm{CY}$ and its metabolites can be a promising accessory tool in anti-tumor therapy.

The mechanism of CY influences macrophage immune function in as was shown previously in case of peritoneal Mf and TMf and seems to be the effect of network of different related factors. The analysis shows as the most important the influence of $\mathrm{CY}$ on the secretory activity of Mf which is the inhibition of IL-10 (in case of peritoneal Mf) and TGF- $\beta$ (mainly in case of TMf) with parallel activation of proinflammatory cytokines secretion mainly IL-6, and to a lesser degree IL-12. Both cytokine signals lead to activation of antigen presentation in Mf. The other important factor mediated by CY treatment is an influence on the activation of a cell surface markers expression responsible for uptaking antigen into APC (FcyR I, FcyRII, CD23 - FceRII/III) and their following presentation to $\mathrm{T}$ lymphocytes subpopulations (CD80/CD86, MHC class II, CD14-LPS receptor).

\subsection{Influence of CY on testicular macrophages}

Although our experiments indicate that in the testis - immune privileged organ - some subpopulations of Mf are potentially able to present antigen if they would sneak through the blood-testis barrier, they also suggest how this potential activity is under control of other Mf and Sertoli cells. Our previous observations have shown that TMf are poor producers of oxygen radicals and nitric oxide both involved in the mechanisms of natural immunity which may be an evolutionary adaptation to diminish the risk of DNA mutations during spermatogenesis. Additionally we showed that specific immune responses controlled by the male gonads minimize the risk of development of autoimmune reactions and are potentially deleterious to testicular functions (Bryniarski 2004). Testicular Mf are good producers of TGF- $\beta$, which allows them to play an important functional population of testicular interstitial tissue cells that preserves state of tolerance in testes an immune privilege organs. 
That state of tolerance eliminates the cellular immune response from the testis and in consequence makes an extremely dangerous the viral infection as well as in malignances taking place in testis. CY treatment often change TMf activity from unresponsiveness into actively antigen presenting cells which in consequence help to undertake anticancer response but also often can leads to activation an autoimmune response and immunological infertility as a consequences of chemotherapy.

\section{Conclusions}

The influence of $\mathrm{CY}$ on Mf can be summarized as a sum of several different mechanisms mediated by macrophages such as secretion of a specific pattern of cytokines and enhances expression of cell surface markers that can stimulate antigen presenting function by macrophages and last not least production of ROI's. On the other side there are several observations that low dose $\mathrm{CY}$ treatment has a direct influence of the different regulatory cells in immune system. One of them is a negative activation of CD8 ${ }^{+} \mathrm{T}$ lymphocytes leading to elimination of their effector mediators - suppressor cytokines secretion mainly TGF- $\beta$ and IL-10, which negatively regulate the cellular immune response, but do not express any negative effect of humoral response. That state of abrogation of unresponsiveness is also observed experimentally when the TNP-substituted Mf obtained from oil-induced donors are injected into $\mathrm{CY}$ treated recipients of cells (see Figure 5) group C. In that case instead of unresponsiveness expressed by control group (group A) strong CHS response appears $24 \mathrm{~h}$ after challenge. This phenomenon clearly shows the influence of $\mathrm{CY}$ on the suppressor network of $\mathrm{T}$ reg cells. This also clearly shows that CY-manipulation leads to manifold effects in which manifold can be described as wiped out or misdirected. In the literature there are two papers suggesting depletion activity of CY on Treg CD4 CD25 T lymphocytes and Treg CD4 CD25 FoxP3+ lymphocytes (Ghiringhelli et al. 2004 \& Zhao et al. 2010)

\begin{tabular}{|c|c|c|c|}
\hline \multirow{2}{*}{ Group } & \multicolumn{2}{|c|}{$\begin{array}{c}\text { Cyclophosphamide treatment of } \\
\text { donors } \\
\text { of TNP-Mf }\end{array}$} & $\begin{array}{c}\text { Contact sensitivity response } \\
\text { of TNP-MIf } \\
\text { (AISE) }\end{array}$ \\
\hline A & - & - & \\
\hline B & + & - & \\
\hline C & - & + & \\
\hline D & + & + & \\
\hline
\end{tabular}

1×106 TNP substituted Mf (groups A and C) or TNP-Mf CY (groups B and D) were injected i.v. into naive (groups A and B) or treated with low dose of CY CBA/J mice. Seven days later the CHS response was measured (see legend to Table 2). Statistical significance (a posteriori Bonferroni test) Group A ve groups B, C and D p $<0.001$.

Fig. 5. Alleviation of suppression of contact sensitivity response induced by low dose treatment with cyclophosphamide applied either to macrophage donors or recipients. 
A single administration of low dose of $\mathrm{CY}(50 \mathrm{mg} / \mathrm{kg})$ into either donors or recipients restores the ability of Mf to induce significant CS reaction as a result of: i.) elimination of suppressive properties of Mf; ii.) and/or depletion of population of regulatory $\mathrm{T}$ cells in recipients or iii.) elimination of their suppressive activities. In vitro studies with metabolites of $\mathrm{CY}$ in contrast to studies in vivo allow identifying the factors which express direct action on selected populations of cells in contrary to experimental research in vivo which is able to identify parallel with direct also indirect effects of cyclophosphamide action on other than macrophages cell populations ( $\mathrm{T}$ reg cells) that may change and modulate the activity of macrophages and their influence on the immune response. We propose the schemes which summarize the influence of low doses of $\mathrm{CY}$ on the immune response in mice (Figures 6a-6c).

Figure 6 a-c. The network of the $\mathrm{CY}$ influence on the macrophage and regulatory $\mathrm{T}$ cells in mice.

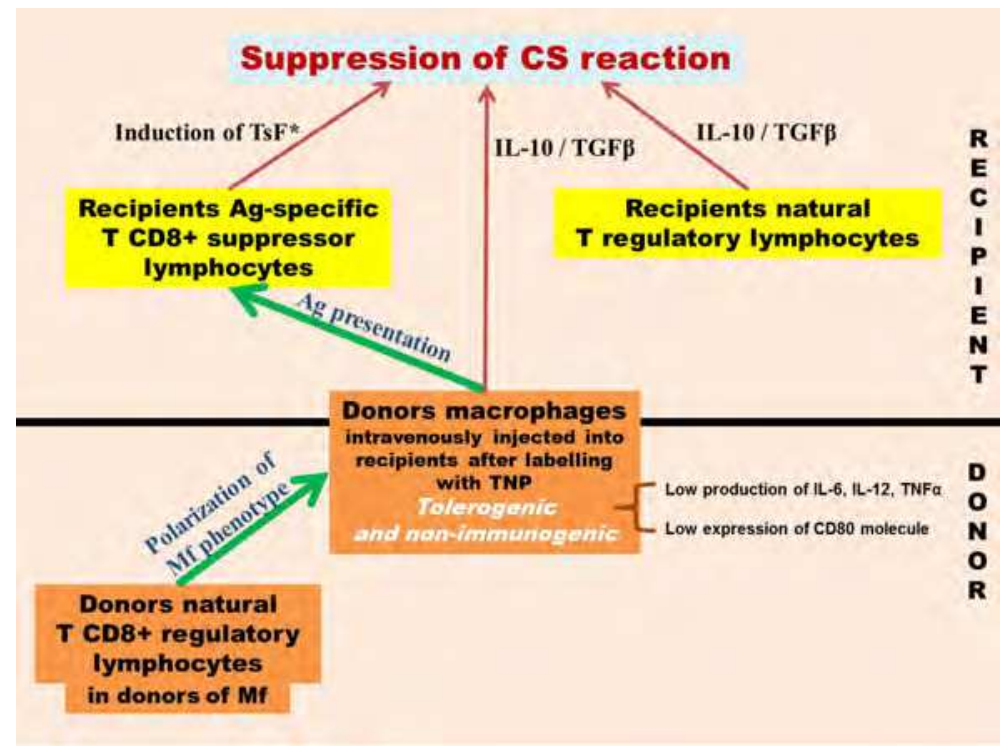

* Induction of T suppressor factor (see the reference by Bryniarski et al. 2-nd European Congress of Immunology Berlin 2009)

Fig. 6.a. Activation of unresponsiveness in recipients after i.v. injection of TNP substituted $\mathrm{Mf}$ - lack of CHS reaction. 


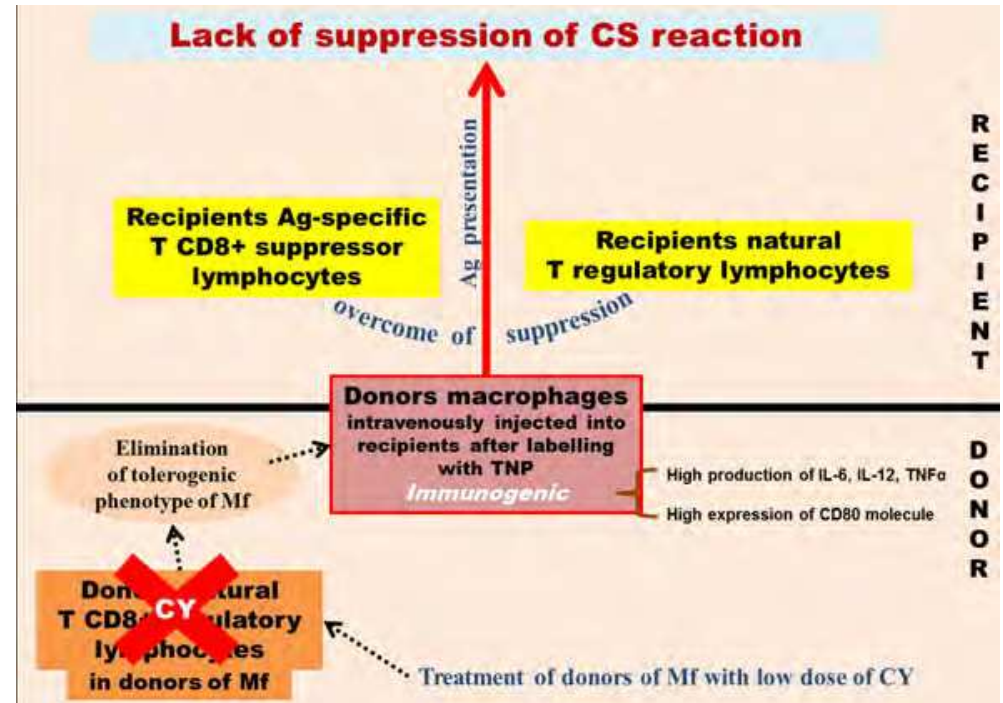

Fig. 6.b. The network of the CY influence on the macrophage and regulatory cells in mice. The i.v. injection into naïve recipient of TNP substituted Mf harvested from CY treated donors results in a state of high CHS reaction $24 \mathrm{~h}$ after challenge.

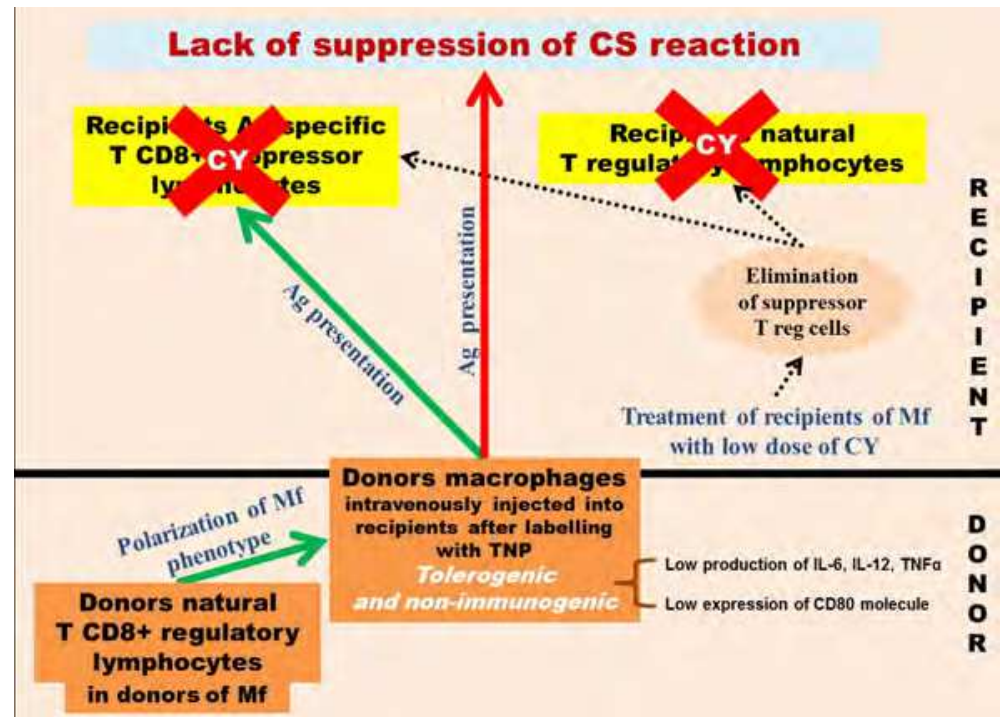

Fig. 6.c. The network of the $\mathrm{CY}$ influence on the macrophage and regulatory cells in mice. The i.v. injection of TNP substituted Mf harvested from naïve donors into recipients previously treated with low dose of CY results in expression of high CHS reaction $24 \mathrm{~h}$ after challenge with PCL hapten. The activation of CHS is the effect of blocking of natural reg $\mathrm{T}$ cells or antigen specific Ts cells. 


\section{Acknowledgments}

The work is supported by grant No K/ZDS/001429 to KB. The author expresses gratitude to Ms. Katarzyna Nazimek M.Sc. for precious help in preparing the Figures and Tables.

\section{References}

Adjalle, R. Plouin, P. F. Pacak, K. \& Lehnert, H. (2009). Treatment of malignant pheochromocytoma. Horm Metabol Res. Vol. 41, pp. 687-696.

Audia, S. Nicolas, A. Cathelin, D Larmonier N, Ferrand C, Foucher P, Fanton A, Bergoin E, Maynadie M, Arnould L, Bateman A, Lorcerie B, Solary E, Chauffert B, Bonnotte B.(2007). Increase of $\mathrm{CD} 4{ }^{+} \mathrm{CD} 25$ high regulatory $\mathrm{T}$ cells in the peripheral blood of patient with metastatic carcinoma: a phase I clinical trial using cyclophosphamide and immunotherapy to eliminate $\mathrm{CD} 4{ }^{+} \mathrm{CD} 25^{+} \mathrm{T}$ lymphocytes. Clinical and Experimental Immunology. Vol. 150, pp. 532-30.

Ben-Efraim, S. (2001). Immunomodulating anticancer alkylating drugs targets and mechanisms of activity. Curr Drug Targ Vol. 2, pp. 197-212.

Breitfeld, P. P. \& Meyer, W. H. (2005). Rhabdomyosarcoma: New windows of opportunity. The Oncologist. Vol. 10, pp. 518-527.

Bryniarski, K. Ptak, M. \& Ptak, W. (1996). The in vivo and in vitro effects of an alkylating agent, mechlormethamine, on IL-6 production in mice and the role of macrophages. Immunopharmacology. Vol. 34, pp. 73-78.

Bryniarski, K. Szczepanik, M. Maresz, K. Ptak, M. \& Ptak, W. (2004). Subpopulations of mouse testicular macrophages and their immunoregulatory function. American Journal of Reproductive Immunology. Vol. 52, pp. 27-35.

Bryniarski, K. Szczepanik, M. Ptak, M. Zemelka, M. \& Ptak, W. (2009). Influence of cyclophosphamide and its metabolic products on the activity of peritoneal macrophages in mice. Pharmacological Reports. Vol. 61, pp. 550-557.

Bryniarski, K. Ptak, M. Sikora, E. Szczepanik, M. Guerrier-Takada, C. Altman, S. Askenase, P.W. Ptak, W. (2009) Role of low molecular weight RNA in contact sensitivity response. Medimond International Proceedings ECI Berlin 2009 pp 183-186.

Burger, R. A. (2007). Experience with Bevacizumab in the management of epithelial ovarian cells. Journal of Clinical Oncology. Vol. 25 (20) Jul.10, pp. 2902-2908.

Cole, M. E. Broaddus, R. Thaker, P. Lauden, C. \& Freedman, R. S. (2008). Placenta - side of trophoblastic tumors: case of resistant pulmonary metastasis. Nature, Clinical Practice, Oncology. Vol. 5, (3), pp. 171-175.

Czajkowska, B. Ptak, M. Bobek, M. Bryniarski, K. \& Szczepanik, M. (1995). Different isoenzyme patterns of nonspecific esterases and the level of IL-6 production as markers of macrophage functions. Folia Histologica et Biologica Vol. 33, (2), pp. 111115.

Durhan, N. Singh, S. Singh Kadin, Y. Durhan, V. Rajotia \& N. Sangwen N. (2009). Primary leiomyosarcoma of broad ligament: case report and review of literature. Arch Gynocol Obstet. Vol. 279, pp. 705-708.

Ghiringhelli, F. Larmounier, N. Schmidt, E. Parcellier, A. Cathelin, D. Garrido, C. Chauffert, B. Solary, E. Bonnotte, B. \& Martin F. (2004). CD4 ${ }^{+} \mathrm{CD} 25^{+}$regulatory T cells suppress tumor immunity but are sensitive to cyclophosphamide which allows 
immunotherapy of established tumors to be curative. European Journal of Immunology. Vol. 34 pp.336-344.

Lerch,M. \& Pichler, W. J. (2004). The immunological and clinical spectrum of delayed druginduced exanthems. Current Opinion in in Allergy and Clinical Immunology. Vol. 4, pp. 411-419.

Luznik, L. \& Fusch, E. J. (2010). High-dose post-transplantation cyclophosphamide to promote graft-host tolerance after allogenic hematopoetic stem cell transplantation. Immunological Research. Vol. 47 (1-3), pp. 65-77.

Marcinkiewicz, J. Bryniarski, K. \& Ptak, W. (1994). Cyclophosphamide uncovers two separate macrophage subpopulations with opposite immunogenic potential and different patterns of monokine production. Cytokine. Vol. 6 (5), pp. 472-477.

Posadas, S. J. \& Pichler, W. J. (2007). Delayed drug hypersensitivity reactions - new concepts. Clinical and Experimental Allergy. Vol. 37, pp. 989-999.

Snowden, J. A. Angel, C. A. Winfield, D. A. Pringle, J. H. West, K. P. (1997). Angiotropic lymphoma: report of a case with histiocytic features. Journal of Clinical Pathology Vol. 50, pp. 67-70.

Suzuki, R. (2010). Treatment of advanced NK/T cell lymphoma: Nasal type and aggressive NK-cell leukemia. Int J Hematol. Vol. 92, pp. 697-701.

Szczepanik, M. Bryniarski, K. Pryjma, J. \& Ptak, W. (1993). Distinct population of antigenpresenting macrophages are required for induction of effector and regulatory cells in contact sensitivity response in mice. Journal of Leukocyte Biology Vol. 53 (3), pp. 320-326.

Vitolo, V. Ferreri, A. J. M. \& Zucca, E. (2008). Primary testicular lymphoma. Critical Review in Oncology/Hematology.Vol.65, pp. 183-189.

Weiner, H. L. and Cohen, J. A. (2002). Treatment of multiple sclerosis with cyclophosphamide: critical review of clinical and immunologic effects. Multiple Sclerosis. Vol. 8, pp. $142-154$.

Zhao, J. Cao, Y. Lei, Z. Yang, Z. Zhang, B. \& Huang B. (2010). Selective depletion of $\mathrm{CD}^{+}{ }^{+} \mathrm{CD} 25^{+} \mathrm{FoxP}^{+}$regulatory $\mathrm{T}$ cells by low-dose cyclophosphamide is explained by reduced intracellular ATP levels. Cancer Research. Vol. 70, (12), pp. 4850-4858. 


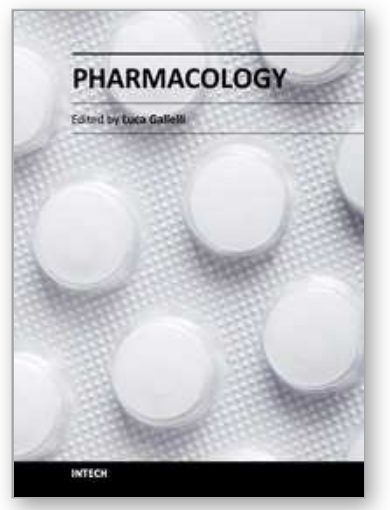

\author{
Pharmacology \\ Edited by Dr. Luca Gallelli
}

ISBN 978-953-51-0222-9

Hard cover, 720 pages

Publisher InTech

Published online 14, March, 2012

Published in print edition March, 2012

The history of pharmacology travels together to history of scientific method and the latest frontiers of pharmacology open a new world in the search of drugs. New technologies and continuing progress in the field of pharmacology has also changed radically the way of designing a new drug. In fact, modern drug discovery is based on deep knowledge of the disease and of both cellular and molecular mechanisms involved in its development. The purpose of this book was to give a new idea from the beginning of the pharmacology, starting from pharmacodynamic and reaching the new field of pharmacogenetic and ethnopharmacology.

\title{
How to reference
}

In order to correctly reference this scholarly work, feel free to copy and paste the following:

Krzysztof Bryniarski (2012). The Influence of Cyclophosphamide on Immune Function of Murine Macrophages, Pharmacology, Dr. Luca Gallelli (Ed.), ISBN: 978-953-51-0222-9, InTech, Available from:

http://www.intechopen.com/books/pharmacology/the-influence-of-cyclophosphamide-on-immune-function-ofmurine-macrophages

\section{INTECH}

open science / open minds

\section{InTech Europe}

University Campus STeP Ri

Slavka Krautzeka 83/A

51000 Rijeka, Croatia

Phone: +385 (51) 770447

Fax: +385 (51) 686166

www.intechopen.com

\section{InTech China}

Unit 405, Office Block, Hotel Equatorial Shanghai

No.65, Yan An Road (West), Shanghai, 200040, China

中国上海市延安西路65号上海国际贵都大饭店办公楼405单元

Phone: +86-21-62489820

Fax: +86-21-62489821 
(C) 2012 The Author(s). Licensee IntechOpen. This is an open access article distributed under the terms of the Creative Commons Attribution 3.0 License, which permits unrestricted use, distribution, and reproduction in any medium, provided the original work is properly cited. 